\title{
Switchable Rashba anisotropy in layered hybrid organic-inorganic perovskite by hybrid improper ferroelectricity
}

\author{
Fei Wang ${ }^{1}$, Heng Gao ${ }^{2,3}$, Coen de Graaf $\mathbb{D}^{1,4 凶}$, Josep M. Poblet ${ }^{1}$, Branton J. Campbell (iD ${ }^{5}$ and Alessandro Stroppa (iD ${ }^{6 凶}$
}

Hybrid organic-inorganic perovskites (HOIPs) are introducing exotic directions in the photovoltaic materials landscape. The coexistence of inversion symmetry breaking and spin-orbit interactions play a key role in their optoelectronic properties. We perform a detailed study on a recently synthesized ferroelectric layered $\mathrm{HOIP}$, (AMP)Pbl 4 (AMP = 4-aminomethyl-piperidinium). The calculated polarization and Rashba parameters are in excellent agreement with experimental values. Moreover, we report a striking effect, i.e., an extraordinarily large Rashba anisotropy that is tunable by ferroelectric polarization: as polarization is reversed, not only the spin texture chirality is inverted, but also the major and minor axes of the Rashba anisotropy ellipse in $k$-space are interchanged - a pseudo rotation. A k.p model Hamiltonian and symmetry-mode analysis reveal a quadrilinear coupling between the cationrotation modes responsible for the Rashba ellipse pseudo-rotation, the framework rotation, and the polarization. These findings may provide different avenues for spin-optoelectronic devices such as spin valves or spin FETs.

npj Computational Materials (2020)6:183; https://doi.org/10.1038/s41524-020-00450-z

\section{INTRODUCTION}

In recent years, there has been an intense research effort surrounding the conversion of solar energy into electric power through photovoltaic cells ${ }^{1-3}$. Hybrid organic-inorganic perovskites (HOIPs) with composition $\mathrm{ABX}_{3}$ ( $\mathrm{A}=$ organic cation, such as $\left[\mathrm{CH}_{3} \mathrm{NH}_{3}\right]^{+} ; \mathrm{B}=$ divalent metal cation, such as $\mathrm{Sn}^{2+}, \mathrm{Pb}^{2+} ; \mathrm{X}=$ halogen anion) are proposed as a next generation of materials for solar cells and light-emitting diodes ${ }^{4-8}$. The increase in structural degrees of freedom from the inclusion of organic cations into a $\mathrm{BX}_{3}$ inorganic framework enables structural distortions and columnar shifts which are otherwise forbidden in standard inorganic perovskites. Furthermore, ferroelectricity (FE) originating from structural symmetry breaking is a potentially critical phenomenon in such soft and flexible photovoltaic materials, where the electric field plays an important role in promoting electron-hole pair separation and suppressing charge recombination, inherently breaking the Shockley-Queisser limit $^{9-13}$. Therefore the FE properties of perovskites have attracted broad attention, though their presence and influence in perovskite solar cells are still a matter of debate ${ }^{14-19}$.

A strong spin-orbit coupling $(\mathrm{SOC})$ involving the heavy $\mathrm{Pb}$ atom and its surrounding halogens, combined with the absence of inversion symmetry in the crystal structure, can lead to a Rashba effect which, in turn, can impact photovoltaic performance ${ }^{20-24}$. Non-centrosymmetry provides a spin-degenerate band with the possibility to split two reversely spin-polarized states described by the equation:

$$
E_{ \pm}(k)=\frac{\hbar^{2} k^{2}}{2 m} \pm a_{R}|k|
$$

which can be used to clarify the dispersion relation of electrons (or/and holes), where $a_{R}$ is the Rashba splitting parameter ${ }^{25-27}$. The coexistence of FE and a Rashba effect is believed to mediate interesting effects, such as the switching of spin-texture chirality with an electric-polarization reversal ${ }^{28,29}$. These effects are mainly studied in the context of inorganic materials; only a few hybridmaterial examples have been explored so $\operatorname{far}^{25,30,31}$.

Very recently, Sum et al. demonstrated the presence of both FE and a Rashba effect in a layered Dion-Jacobson (DJ) phase HOIP with formula (AMP)Pbl ${ }_{4}$, where AMP is the divalent 4aminomethyl-piperidinium cation (Fig. 1, FE state) ${ }^{32}$. Its measured value of spontaneous polarization $\left(P_{\mathrm{s}}\right)$ is $9.8 \mu \mathrm{C} \mathrm{cm}^{-2}$, which is very high among $\mathrm{HOIPs}^{33,34}$, and more comparable to those of conventional inorganic perovskites such as $\mathrm{BaTiO}_{3}(15-26 \mu \mathrm{C}$ $\left.\mathrm{cm}^{-2}\right)^{35,36}$. A measured Rashba splitting energy $\left(E_{R}\right)$ of $85 \mathrm{meV}$ and Rashba coefficient $\left(a_{R}\right)$ of $2.60 \mathrm{eV} \AA$ suggest real promise for spintronic applications ${ }^{30,31}$. This exciting experimental evidence now motivates a theoretical investigation into the microscopic mechanism of FE and its interplay with SOC in the HOIPs.

Starting with this aim in mind, we perform a detailed density functional theory (DFT) analysis of (AMP)Pbl ${ }_{4}$, focusing on estimates of the ferroelectric and spin-orbit properties of the band structure. While the estimate of the Rashba splitting is now routine work, we focus here on the corresponding anisotropy, which is far much less studied. Our estimate of $P_{s}$ and $a_{R}$ are $10.72 \mu \mathrm{Ccm}^{-2}$ and $2.39 \mathrm{eV} \AA$, in excellent agreement with the experimental $9.80 \mu \mathrm{C} \mathrm{cm}^{-2}$ and $2.60 \mathrm{eV} \AA$. In addition, the $E_{R}$ of spin-polarized bands is calculated to be $16 \mathrm{meV}$, which is of the same order of magnitude as the experimental $85 \mathrm{meV}$ value. In addition to a spin-texture that can be switched via ferroelectric polarization reversal, we further observe a sizeable anisotropy in the Rashba spin splitting. Remarkably, we show that the axis of major anisotropy can be switched between two orthogonal directions by reversing the ferroelectric polarization from $+P$ to $-P$. Reversing the FE polarization induces an apparent $90^{\circ}$

${ }^{1}$ Departament de Química Física i Inorganica, Universitat Rovira i Virgili, c/Marcel.lí Domingo 1, Tarragona 43007, Spain. ${ }^{2}$ Beijing National Laboratory for Condensed Matter Physics, Institute of Physics, Chinese Academy of Sciences, Beijing 100190, China. ${ }^{3}$ Songshan Lake Materials Laboratory, Dongguan, Guangdong 523808, China. ${ }^{4}$ ICREA, Pg. Lluis Companys 23, Barcelona 08010, Spain. ${ }^{5}$ Department of Physics \& Astronomy, Brigham Young University, Provo, UT 84602, USA. ${ }^{6}$ CNR-SPIN c/o Department of Physical and Chemical Sciences, University of L'Aquila, Via Vetoio, I-67100 Coppito, L'Aquila, Italy. ${ }^{凶}$ email: coen.degraaf@urv.cat; alessandro.stroppa@spin.cnr.it 
a)

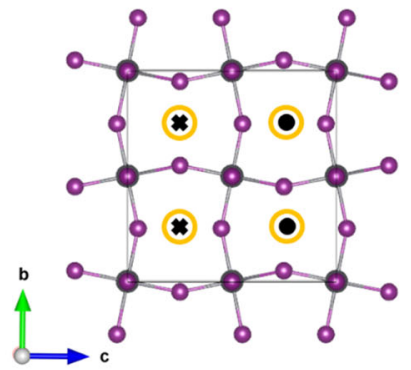

b)

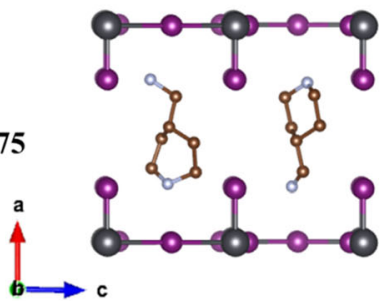

c)

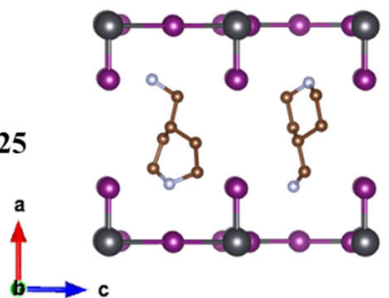

AFE states

$(\lambda=0)$
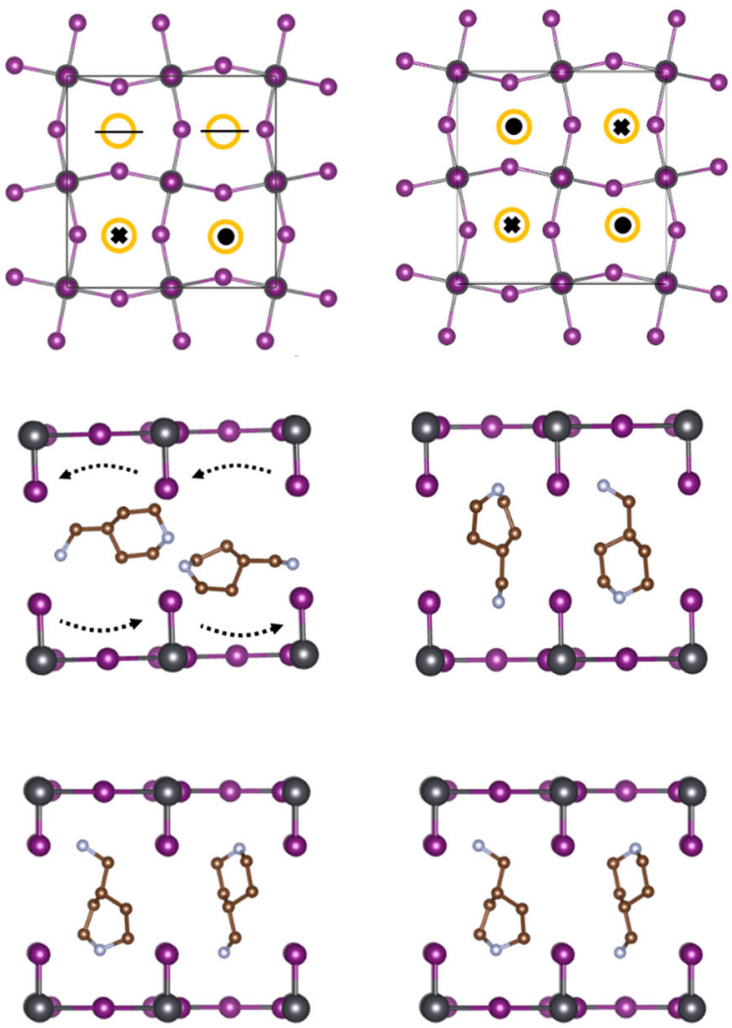

intermediate state

$(\lambda=0.5)$

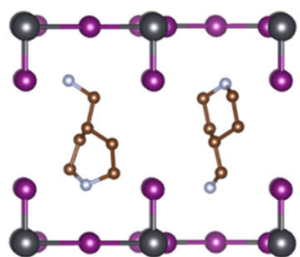

FE state

$(\lambda=1)$

Fig. 1 The structures of AFE and FE $(+\boldsymbol{P})$ phases. Top $(\mathbf{a})$ and side $(\mathbf{b}, \mathbf{c})$ views with purple Pb, gray I, brown $\mathrm{C}$, and blue N. AMP-cation hydrogen atoms are omitted for clarity. The black dot or cross in the yellow circle of (a) represent the AMP-cation orientation as having the aminomethyl group of the molecule along with the up $(+a)$ or down $(-a)$ axis, respectively.

"rotation" of the anisotropy ellipse, i.e., a pseudo-rotation. This effect has never been highlighted in the context of usual spintexture tunability under polarization reversal and may suggest interesting avenues for spin-optoelectronic devices based on HOIPs.

\section{RESULTS AND DISCUSSION}

Structural analysis

We start our simulations with the experimental ferroelectric phase of (AMP) $\mathrm{Pbl}_{4}$ refined at $T=298 \mathrm{~K}$ having space group $P C\left(\# 7, C_{s}^{2}\right)$ with monoclinic $b$-axis in ref. ${ }^{32}$. Two nearby $\mathrm{AMP}^{2+}$ cations located in the space between $\left[\mathrm{Pbl}_{4}\right]^{2-}$ layers have alternating orientations when they are adjacent along the $b$ or $c$ axes, but common orientations when adjacent along with diagonal $b+c$ or $b-c$ directions, as shown in Fig. 1 (FE state), so that the structure lacks a center of inversion, thus paving the way for the existence of ferroelectric polarization in the system. It is interesting to note that the cation's center of mass is displaced somewhat from the ideal midpoint between two adjacent $\left[\mathrm{Pbl}_{4}\right]^{2-}$ layers so as to be slightly closer to one layer or the other. We perform quantum chemical analyses of the isolated $\mathrm{AMP}^{2+}$ cation using both the Hirshfield ${ }^{37}$ and Natural population methods ${ }^{38}$ implemented in the Gaussian 16 software ${ }^{39}$. Both methods define an electric dipole moment of the molecule pointing approximately towards the aminomethyl group from the center of the AMP cation, as shown in Supplementary Fig. 2. Although the long-range ordering of AMP-cation orientations should be associated with a strong FE polarization along the $a$-axis at the first glance, this alone is not sufficient for understanding the overall polarization of a hybrid system, which typically has several different contributions to the polarization, as explained in details in ref. ${ }^{32}$. According to the modern theory of polarization ${ }^{40}$, we introduce a centrosymmetric anti-ferroelectric (AFE) phase by enforcing the existence of an inversion point and define a suitable path connecting the AFE and FE state via atomic rotations or/and displacements in order to estimate the polarization by first-principles calculations. The AFE model is built from the FE model by simultaneously rotating two $\mathrm{AMP}^{2+}$ cations adjacent along the $b$-axis by $180^{\circ}$ so that the diagonal AMP cations are related by inversion symmetry. The $\left[\mathrm{Pbl}_{4}\right]^{2-}$ the framework is also properly centrosymmetric to ensure that the AFE structure (both framework and AMP cations) possesses an inversion symmetry point at $(0.5,0.5,0.5)$, as shown in Fig. 1 (AFE state). The resulting space group symmetry is $P 2 / \mathrm{m}$ $\left(\# 10, C_{2 h}^{1}\right)$ with a monoclinic $b$-axis in the same unit cell.

\section{Ferroelectric properties}

We introduce the normalized variable $\lambda$ to simultaneously parameterize both the rotations of the AMP cations and the atomic displacements of the $\left[\mathrm{Pbl}_{4}\right]^{2-}$ framework from AFE $(\lambda=0)$ to FE $(\lambda=1)$ states. Therefore $\lambda$ represents the amplitude of the combined roto-displacive distortions connecting the prototype AFE-phase structure and the real FE-phase structure. The intermediate structures along the path $(0 \leq \lambda \leq 1)$ are only introduced in order to monitor the accidental introduction of a quantum of polarization and to ensure a continuous variation of the polarization along the path itself. In practice, $\lambda$ has been varied infinite increments, so that stepwise AMP-cation rotations are 


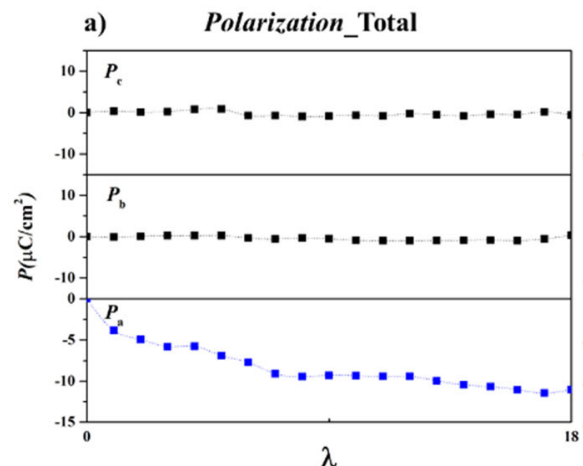

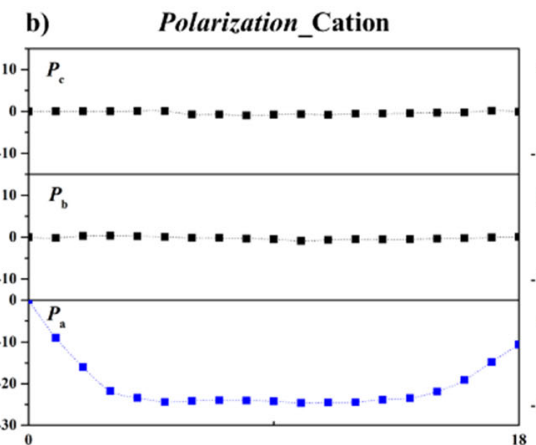

$\lambda$ c) Polarization_Framework

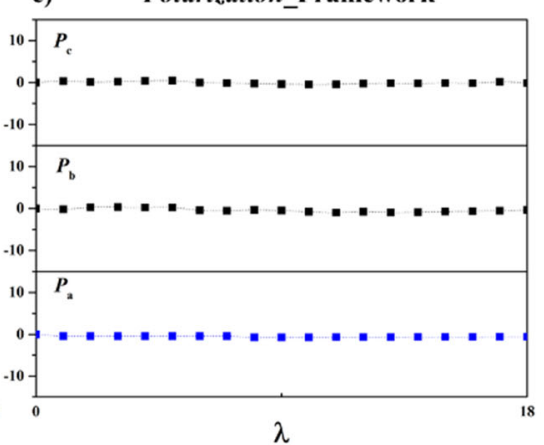

Fig. 2 The FE polarization along the $a-, b$-, and $c$-axes of (AMP)Pbl ${ }_{4}$ as a function of the parameter $\lambda$, which represents the normalized $\mathbf{A M P}^{2+}$ cation rotations and $\left[\mathrm{Pbl}_{4}\right]^{2-}$ framework displacements. a The total FE polarization. $\mathbf{b}, \mathbf{c}$ The polarization contribution of the cation and framework, respectively.

approximated by 18 steps of $10^{\circ}$ each from $0^{\circ}$ (AFE state) to $180^{\circ}$ (FE state), together with incremental linear displacements of the $\left[\mathrm{Pbl}_{4}\right]^{2-}$ framework.

In order to understand the origin of the polarization, the separate contributions of the organic $\mathrm{AMP}^{2+}$ cations and the inorganic $\left[\mathrm{Pbl}_{4}\right]^{2-}$ a framework to the total polarization are considered in three different ways. Firstly, we rotate AMP cations while keeping the $\left[\mathrm{Pbl}_{4}\right]^{2-}$ framework centrosymmetric. In this way, we obtain the contribution coming from the organic groups $\left(P_{\text {cation }}\right)$, i.e., a rotational contribution to the polarization. Secondly, the displacive distortions of $\left[\mathrm{Pbl}_{4}\right]^{2-}$ framework are considered while fixing AMP cations in a centrosymmetric configuration. In this way, the polarization only originates from the inorganic framework $\left(P_{\text {frame }}\right)$, i.e., a displacive contribution to the total polarization. Finally, both AMP-cation rotations and $\left[\mathrm{Pbl}_{4}\right]^{2-}$ layer displacements are simultaneously activated in order to recover the total polarization $\left(P_{\text {total }}\right)$, i.e., the full roto-displacive contribution to the polarization. In this way, we are able to disentangle the different contributions to the polarization and to observe the couplings between them. For each case, we consider the contribution from the ionic and electronic subsystems to the resulting polarization.

The results of the polarization calculations are presented in Fig. 2. In the AFE state, the net polarization is exactly zero, as expected. As $\lambda$ gradually increases toward 1, the asymmetry with respect to the centric phase and the magnitude of the total polarization both increase steadily along the $a$-axis but remain near zero for the $b$ - and $c$-axis components. For $(\lambda=1)$, we extract the estimated value of $P_{\text {total, }}$ which is equal to $10.72 \mu \mathrm{C}$ $\mathrm{cm}^{-2}$, in very good agreement with the experimental value of $9.80 \mu \mathrm{Ccm}^{-2}$. From our analysis, the contributions from organic cations and inorganic frameworks are $10.34 \mu \mathrm{C} \mathrm{cm}^{-2}$ and $0.59 \mu \mathrm{C} \mathrm{cm}^{-2}$, respectively. Clearly, the AMP cations dominate the total polarization, so that the framework contribution appears negligible in comparison. An applied electric field then mainly rotates the AMP cations while displacing the $\left[\mathrm{Pbl}_{4}\right]^{2-}$ framework only a little. Because the switchable FE polarization is primarily due to the long-range orientational order of the organic cations, the true AFE state lying between the $+P$ and $-P$ states could be consistent with either ordered or disordered but balanced arrangements of AMP-cation orientations, thus supporting a null electric polarization. Indeed, the AFE ordered state of the crystal represents only a computational reference phase to evaluate the final polarization, and in principle may be not exclusively defined. In the present case, we have fixed a possible AFE ordering, but, according to the modern theory of polarization, the final polarization does not depend on the particular path considered for $0 \leq \lambda \leq 1^{41}$.
Electronic structure and Rashba SOC effect

We now examine the electronic structure and Rashba SOC effect of ferroelectric (AMP)Pbl ${ }_{4}$. The Brillouin zone (BZ) and corresponding Rashba spin-polarized bands of the FE structure are shown in Fig. $3 a, b$. The valence band maximum (VBM), conduction band minimum (CBM), and related bandgap are localized in $k$-space around the high-symmetry B point. The VBM and CBM are strongly spin-split due to the spin-orbit interaction and are also shifted away from the B point in $k$-space. As usual, the "momentum offset" $\left(k_{R}\right)$ corresponds to the distance between the apex of the splitting band and the high-symmetry point in $k$-space, while $E_{R}$ corresponds to the energy difference between them. In our case, $k_{R}$ and $E_{R}$ are estimated as $0.0133 \AA^{-1}$ and $16 \mathrm{meV}$ for the VBM and as $0.0132 \AA^{-1}$ and $12 \mathrm{meV}$ for the CBM, which shows rather fairly good agreement with the respective experimental values of $0.067 \AA^{-1}$ and $85 \mathrm{meV}$. Indeed, according to the definition

$a_{R}=\frac{2 E_{R}}{k_{R}}$,

$a_{R}$ is calculated to be $2.39 \mathrm{eV} \AA$ for the VBM, which agrees with the experimental $2.60 \mathrm{eV} \AA$ very well. It must be noted that the $k_{R}$ values for the VBM and CBM are very similar, indicating that they are located essentially at the same point in $k$-space to form a "direct" bandgap, which is beneficial for electronic transitions in photovoltaic-cell applications.

We show the band dispersion of the spin-split bands, i.e., a 3D plot of the VBs around the high-symmetry B point, in Fig. 3c. The corresponding spin-textures of the spin-split bands are projected onto the 2D plane perpendicular to the FE polarization $(P)$ in Fig. $3 d$. In the inner and outer bands, the sense of rotation (lefthanded or right-handed) of the spin-textures are opposite due to the spin-orbit interaction. As reported in refs. ${ }^{42,43}$, the polarization reversal resulting from the application of an external electric field should be able to switch the spin textures. Indeed, we also observe that the spin textures completely switch from left- to a right-handed sense of rotation as the polarization goes from $-P$ to $+P$, as shown in Fig. $3 \mathrm{~d}$, e.

While most studies on spin-orbit related properties estimate the Rashba parameter for a given direction (in the plane perpendicular to the polarization) and visualize the spin-texture and its switching properties, in addition here we focus on the anisotropy of the Rashba parameter and its relationship to the switching of the ferroelectric polarization. To probe this anisotropy, the band structure was calculated on a fine grid of points in the vicinity of the reciprocal-space $B$ point within the $2 \mathrm{D}$ plane perpendicular to the FE polarization (the yellow $k_{y} k_{z}$ plane in Fig. $3 a$ ). The calculated $a_{R}$ parameters are summarized in Fig. $4 a$, where we use a polar plot indicating the blue curves corresponding to the $a_{R}$ obtained from the VBs and the green curves corresponding to those from 


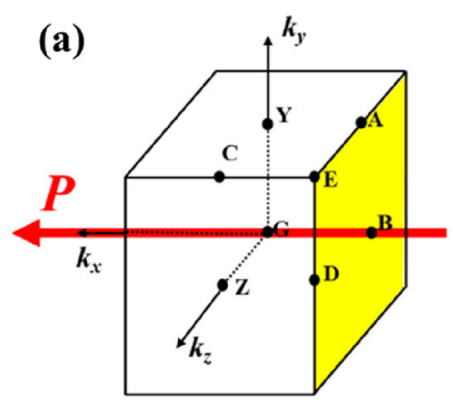

(c)

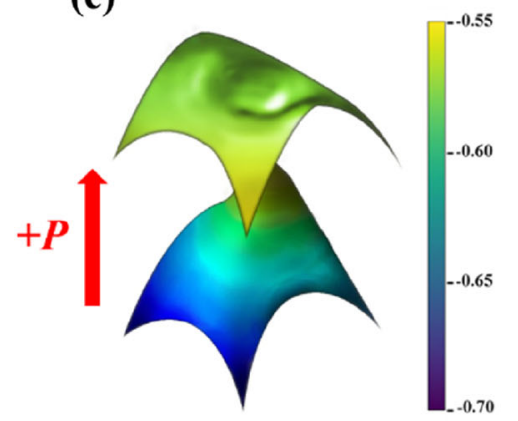

(e)

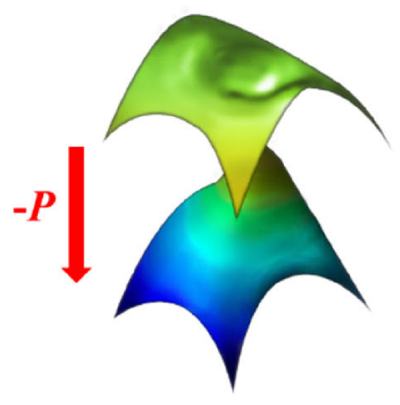

(b)

(d)
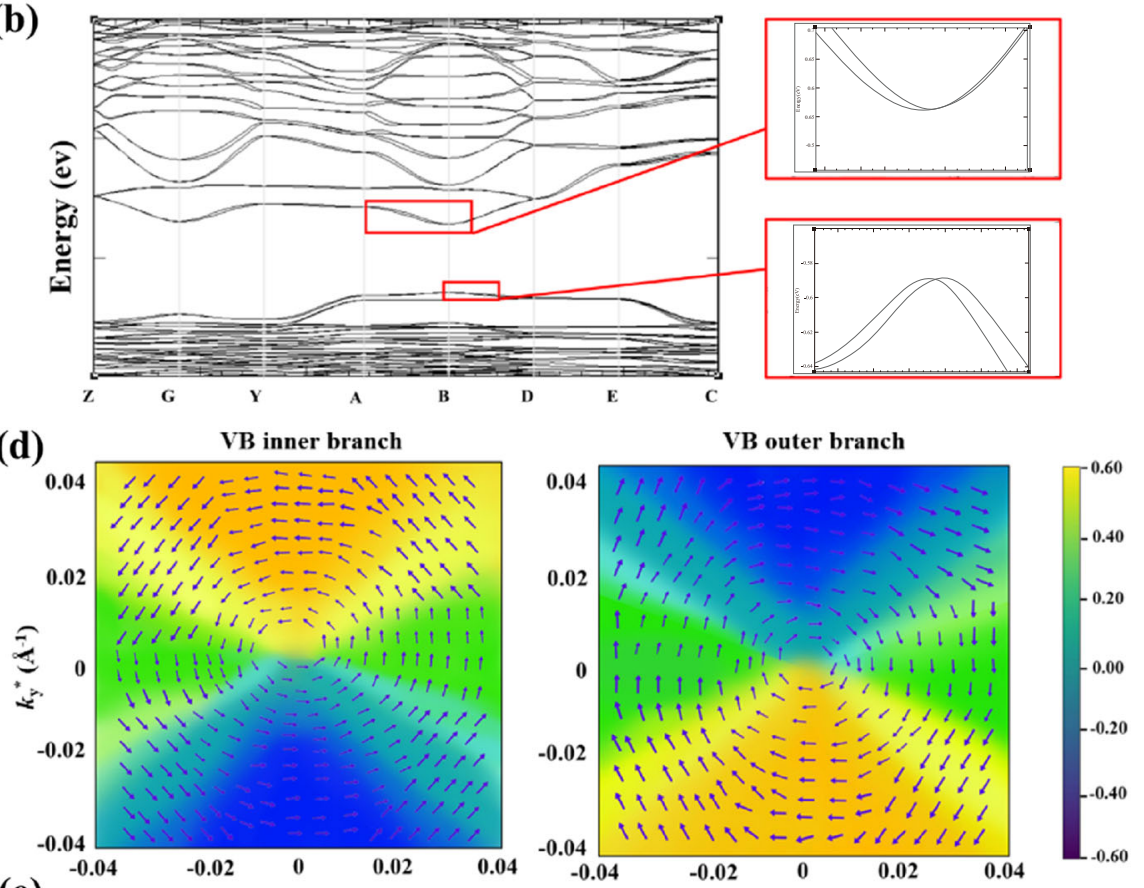
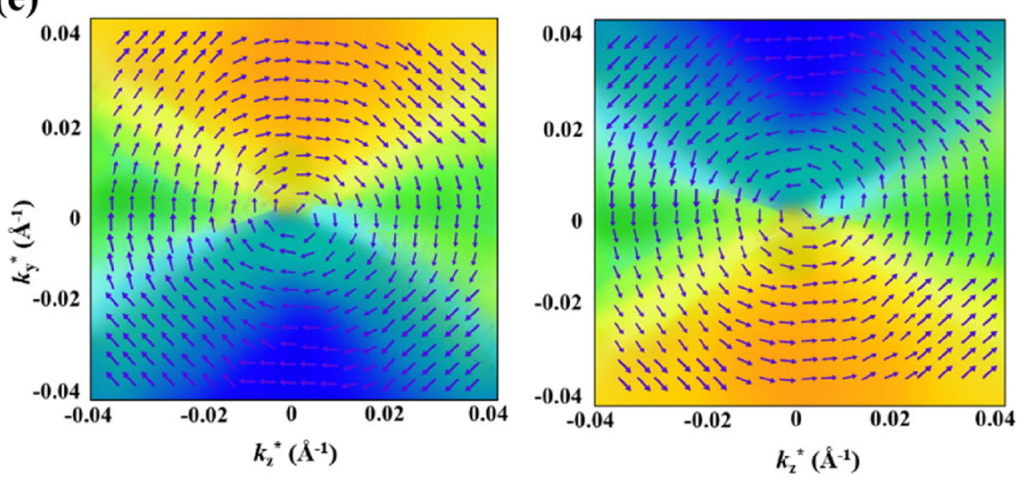

Fig. 3 The band structure of FE state. a The high-symmetry points of BZ. b Dispersed band structures along the whole BZ; the spin-split bands of the VBM (bottom) and CBM (top) were magnified in the red frames for emphasis. c 3D plot of band dispersion for valence bands (VBs) around B point. The color evolution from blue to yellow represents the VB eigenvalues. $\mathbf{d}$, e The spin-textures projected on the 2D plane perpendicular to the FE polarization $(P)$. The color code represents the $s_{X}$ spin component.

the CBs. It can be seen that $a_{R}$ parameters show a marked anisotropy, both at VBs and CBs. The maximum $a_{R}$ of the VB at the B point (the blue curve in Fig. $4 \mathrm{a}$ ) is $2.39 \mathrm{eV} \AA$, located on the $k_{z^{-}}$ axis, whereas the minimum is $0.94 \mathrm{eV} \AA$, located on the $k_{y}$-axis, which indicates that $a_{R}$ is not a constant but rather a function of the angle $\theta$ with respect to the negative $k_{z}$-axis (in the yellow plane in Fig. 3a).

Remarkably, we find that when the FE polarization is switched from $+P$ to $-P$, the major and minor axis of the Rashba anisotropy are exchanged as shown in Fig. $4 \mathrm{~b}$, so that the maximum $a_{R}$ value of the VB (2.22 eV $\AA$ ) shifts from the $k_{z}$-axis (b-axis) to the $k_{y}$-axis ( $c$ axis), and the minimum $a_{R}$ value $(0.87 \mathrm{eV} \AA)$ shifts the opposite way. In other words, while the polarization switches from $+P$ to $-P$, the major axis $a_{R}$ value decreases, while the minor axis $a_{R}$ value increases. If we compare the $+P$ and $-P$ anisotropy ellipses, the net effect is an apparent $90^{\circ}$ rotation of the anisotropy ellipse, i.e., a pseudo-rotation. To the best of our knowledge, this is the first time that a polarization-switchable Rashba anisotropy has been observed, which is also connected to a very large anisotropy.

We propose a structural mechanism for this highly unusual polarization-switchable pseudo rotation of the Rashba anisotropy. As the AMP-cation orientations evolve between the $+P$ and $-P$ states, the roughly $180^{\circ}$ rotation (approximately around the $b$-axis) of each cation not only switches the aminomethyl group between the $+a$ and $-a$ sides of the cation but also rotates its maximumelectron-density plane (MEDP) by about $90^{\circ}$ in the $b c$ plane, which is dual to the $k_{y} k_{z}$ plane of the BZ (can be seen in Supplementary Fig. 3). To verify our hypothesis that this polarization-reversalinduced $90^{\circ}$ rotation of the AMP cation's MEDP is responsible for the rotation of the Rashba-anisotropy, we construct an alternative $-P^{\prime}$ structure from the $+P$ structure by rotating each AMP cations by $180^{\circ}$ around the axis perpendicular to its MEDP, so that its aminomethyl group is still moved between the $+a$ and $-a$ sides, but without allowing the MEDP to rotate in the $b c$ plane. When the structure is thus evolved, we see no rotation of the Rashba anisotropy in its band structures (Fig. 4c, f). Furthermore, compared to the isoenergetic $+P$ and normal $-P$ structures, the alternative $-P^{\prime}$ structure has a $0.48 \mathrm{eV}$ higher energy than $-P$ and is separated from $+P$ by an energy barrier of greater than $1.20 \mathrm{eV}$, demonstrating that the $-P^{\prime}$ structure would be very difficult to obtain at room temperature.

To understand the Rashba anisotropy and spin texture under polarization switching, we construct the effective $k \cdot p$ model near the $B$ point. The little group of the B point is Cs with only mirror 
a)
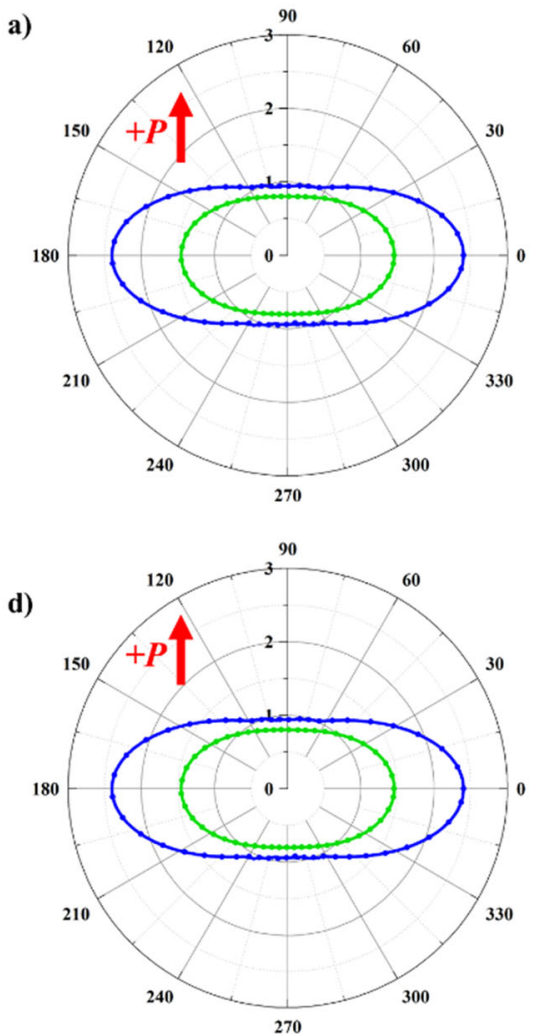

b)
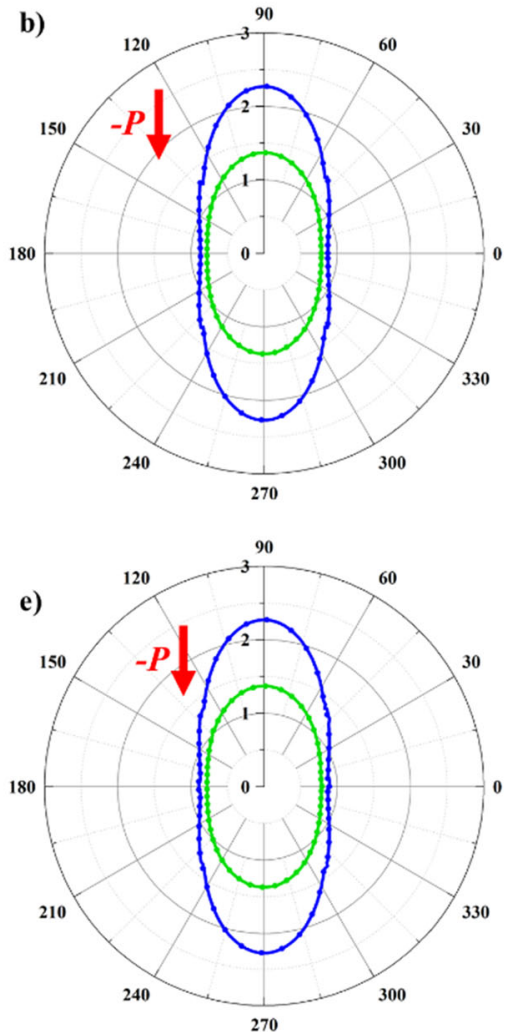

c)
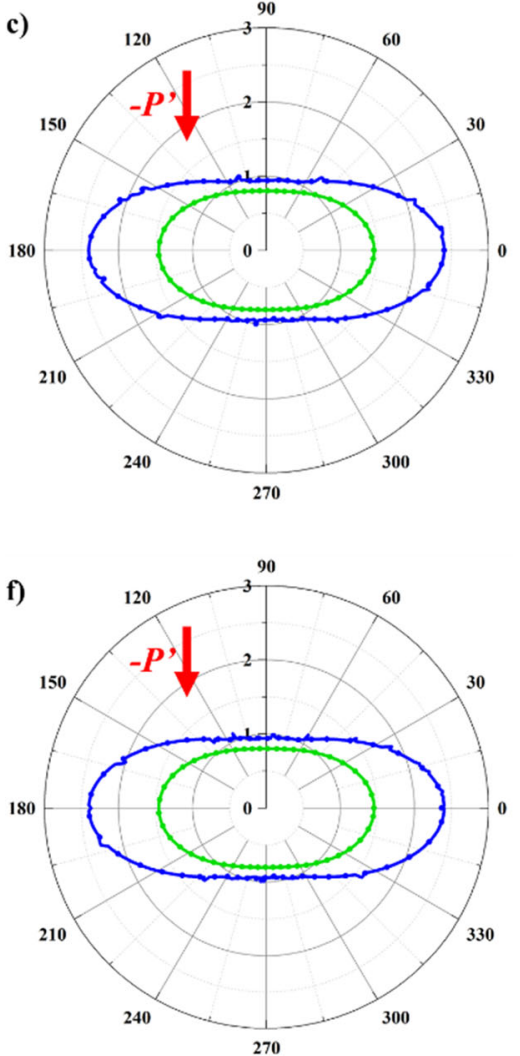

Fig. 4 Polar plots of variable $a_{R}$ for Rashba spin-split bands obtained along with different directions in the plane perpendicular to the FE polarization. The plots of $\mathbf{a}-\mathbf{c}$ are acquired at the $\mathrm{B}$ point while those of $\mathbf{d}$, $\mathbf{e}$ are at the $\mathrm{G}$ point. The blue curves correspond to the $a_{R}$ obtained from the VBs while the green curve corresponds to those from the CBs. The red arrows indicate the direction of the polarization, and $-P^{\prime}$ indicates an alternative $-P$ structure wherein each AMP molecule rotates $180^{\circ}$ relative to the $+P$ structure around the axis perpendicular to its maximum-electron-density plane rather than around the $b$ axis. However, this $-P^{\prime}$ is energetically unfavorable as discussed in the text.

symmetry operator My. Under the mirror transformation My: $(x, y, z) \rightarrow(x,-y, z),\left(k_{x}, k_{y}, k_{z}\right) \rightarrow\left(k_{x},-k_{y}, k_{z}\right) \quad$ and $\left(\sigma_{x}, \sigma_{y}, \sigma_{z}\right) \rightarrow\left(-\sigma_{x}, \sigma_{y},-\sigma_{z}\right)$. Then the two-band Hamiltonian for the VBM or CBM at $k_{x}=0$ near the B point can be written as

$$
H\left(k_{y}, k_{z}\right)=\varepsilon_{0}+\frac{\hbar^{2} k_{y}^{2}}{2 m_{y}}+\frac{\hbar^{2} k_{z}^{2}}{2 m_{z}}+a_{1} k_{z} \sigma_{y}+a_{2} k_{y} \sigma_{z}+a_{3} k_{y} \sigma_{x}
$$

Here $m_{y}$ and $m_{z}$ are the effective masses along the $k_{y}$ and $k_{z}$ directions near the $\mathrm{B}$ point, and $\sigma_{x}, \sigma_{y}$, and $\sigma_{z}$ are Pauli matrices. $a_{1}$, $a_{2}$, and $a_{3}$ are three Rashba parameters. The last three terms are SOC terms that include the Rashba and Dresselhaus SOC effects, which can be rewritten in the form

$$
H_{s o}=\lambda_{D}\left(k_{z} \sigma_{y}+k_{y} \sigma_{z}\right)+\lambda_{R}\left(k_{z} \sigma_{y}-k_{y} \sigma_{z}\right)+a_{3} k_{y} \sigma_{x},
$$

where

$$
\lambda_{D}=\left(a_{1}+a_{2}\right) / 2 \text {, }
$$

and

$$
\lambda_{R}=\left(a_{1}-a_{2}\right) / 2,
$$

are the Dresselhaus and Rashba parameters, respectively ${ }^{44,45}$. The last term $k_{y} \sigma_{x}$ leads to the emergence of the $s_{x}$ spin component on the $k_{y} k_{z}$ plane which is consistent with our first-principles calculation of the spin textures. The eigenvalues of the twoband Hamiltonian reads as

$$
E\left(k_{y}, k_{z}\right)=\varepsilon_{0}+\frac{\hbar^{2} k_{y}^{2}}{2 m_{y}}+\frac{\hbar^{2} k_{z}^{2}}{2 m_{z}} \pm \sqrt{a_{1}^{2} k_{z}^{2}+a_{2}^{2} k_{y}^{2}+a_{3}^{2} k_{y}^{2}} .
$$

To understand the Rashba anisotropy under polarization switching, the eigenvalues can be written as

$$
E\left(k_{r}, \theta\right)=\varepsilon_{0}+\left(\frac{\hbar^{2}}{2 m_{y}} \sin ^{2} \theta+\frac{\hbar^{2}}{2 m_{z}} \cos ^{2} \theta\right) k_{r}^{2} \pm \sqrt{a_{1}^{2} \cos ^{2} \theta+\left(a_{2}^{2}+a_{3}^{2}\right) \sin ^{2} \theta}\left|k_{r}\right|,
$$

in polar coordinates. Here $k_{r}$ is defined as the distance of $k$ from the B point in the $k_{y} k_{z}$ plane, and $\theta$ is the angle between $\vec{k}_{r}$ and $\vec{k}_{z}$. One can define the general "Rashba parameter" with different directions on the $k_{y} k_{z}$ plane in polar coordinates as

$$
a^{\uparrow}(\theta)=\sqrt{a_{1}^{2} \cos ^{2} \theta+\left(a_{2}^{2}+a_{3}^{2}\right) \sin ^{2} \theta},
$$

where the arrow $\uparrow$ means the polarization along with the $+x$ direction corresponding to the $+P$ state. The reorientation of the AMP cation, which involves the combination of a $M_{x}$ mirror operation and a $C_{4 x}$ rotation operation, effects a $90^{\circ}$ rotation of the crystal fields at the cation site such that $(x, y, z) \rightarrow(-x, z, y)$ and $\left(k_{x}, k_{y}, k_{z}\right) \rightarrow\left(-k_{x}, k_{z}, k_{y}\right)$. The eigenvalue of effective Hamiltonian of the $-P$ state should then be

$$
E^{\downarrow}\left(k_{r}, \theta\right)=\varepsilon_{0}+\left(\frac{\hbar^{2}}{2 m_{y}} \cos ^{2} \theta+\frac{\hbar^{2}}{2 m_{z}} \sin ^{2} \theta\right) k_{r}^{2} \pm \sqrt{a_{1}^{2} \sin ^{2} \theta+\left(a_{2}^{2}+a_{3}^{2}\right) \cos ^{2} \theta}\left|k_{r}\right|,
$$

in polar coordinates. So the anisotropy of the Rashba parameter of the $-P$ state is

$$
a^{\downarrow}(\theta)=\sqrt{a_{1}^{2} \sin ^{2} \theta+\left(a_{2}^{2}+a_{3}^{2}\right) \cos ^{2} \theta},
$$

where the arrow $\downarrow$ means the polarization along the $-x$ direction. It is easy to see that

$$
a^{\uparrow}(\theta)=a^{\downarrow}\left(\theta+\frac{\pi}{2}\right) \text {, }
$$


a)

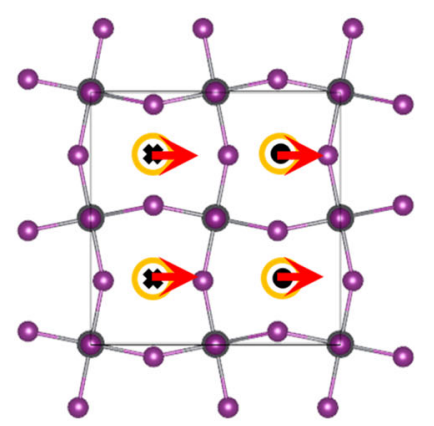

c)

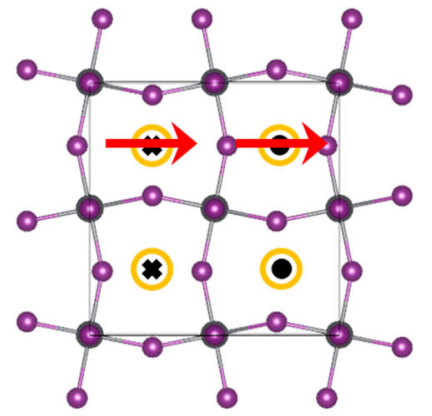

b)

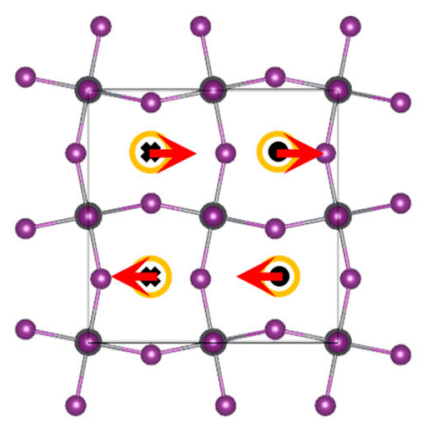

d)

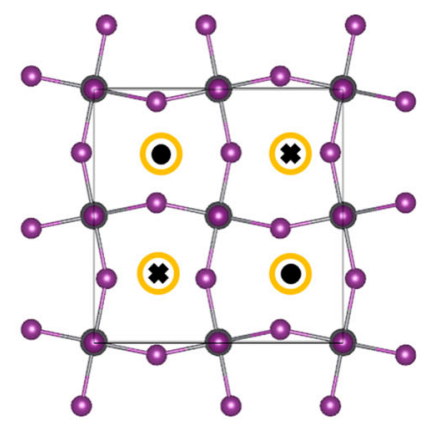

Fig. 5 Molecular rotation modes. Short $\left(90^{\circ}\right)$ and long $\left(180^{\circ}\right)$ red arrows indicate right-handed molecular rotations relative to the AFE configuration. Summing the ferrorotational (FR) (a) and anti-ferrorotational (AFR) (b) patterns of $90^{\circ}$ rotations cause only two of four molecules to rotate by $180^{\circ}$ (c), which is precisely the FE structure (d).

which means the anisotropy of the Rashba parameter is rotated by $90^{\circ}$ under polarization switching, which is equivalent to a $180^{\circ}$ rotation of polarization. The proposed $k \cdot p$ model is thus nicely consistent with our DFT results in terms of a $90^{\circ}$ pseudo-rotation.

We introduce parameter

$\mu=\left|\frac{a_{R}\left(k_{z}\right)-a_{R}\left(k_{y}\right)}{a_{R}\left(k_{y}\right)}\right|=\left|\frac{a_{R}\left(k_{z}\right)}{a_{R}\left(k_{y}\right)}-1\right|$,

to quantify the strength of the anisotropy where $a_{R}\left(k_{z}\right)$ and $a_{R}\left(k_{y}\right)$ are the numerical values of the Rashba parameter along the $k_{z}$ and $k_{y}$ axes of the $\mathrm{BZ}$, respectively. The larger the value of $\mu$, the more pronounced the anisotropy of $a_{R}$ is. In the case of the $-P$ and $+P$ polarizations, $\mu$ has quite similar values of 1.7 and 1.6 , respectively, reflecting the robustness of the Rashba anisotropy in this system.

It is interesting to note that the pseudo-rotation of the Rashba anisotropy induced by polarization reversal occurs not only at a single point in $k$-space, but at several points where the Rashba splitting is effective. Our observations of the Rashba anisotropy in the $V B$ and $C B$ near both the $B$ and $G$ points of the $B Z$ are all qualitatively consistent with the description and explanation of Fig. $4 d-f$ above. At both of these points, the pseudo-rotation of the anisotropy ellipse occurs when reversing the polarization perpendicular to the framework layers.

Symmetry mode analysis

The $+P$ and $-P$ FE structures can be viewed as distorted "children" of an idealized "parent" structure, which allows us to characterize their symmetry-breaking structural variables (i.e., symmetry modes) in terms of the irreducible representations (irreps) of the parent space-group symmetry. We construct this idealized parent from the centrosymmetric AFE structure in the left panel of Fig. 1a by regularizing the $\mathrm{Pbl}_{6}$ octahedra and zeroing the $\mathrm{Pbl}_{6}$ octahedral rotations in order to straighten out the framework, and by replacing each AMP cation with a polar vector (attached to a dummy atom at the molecular centroid location) that points along the appropriate $\pm a$ direction, resulting in centrosymmetric space group Pcmm (\#51, $\left.D_{2 h}^{5}\right)$ on an orthorhombic $(\boldsymbol{a}, \boldsymbol{b} / 2, \boldsymbol{c})$ unit-cell basis with zero origin shift relative to the child. We also idealize the FE children by replacing each AMP cation with an appropriate polar vector in order to apply the symmetry mode analysis consistently.

The key order parameters of the idealized parent structure that characterize our FE child structures are (1) the $\mathrm{Pbl}_{6}$ octahedral framework rotation around the $a$-axis, which belongs to parent irrep $Y_{3}^{+},(2)$ the ferroelectric displacements along the $a$-axis, which belong to FE parent irrep $\Gamma_{2}^{-}$, and (3) the large AMP-cation rotations around the $b$-axis, which are achieved through the cooperative action of two-parent irreps, ferrorotational (FR) irrep $\Gamma_{4}^{+}$and anti-FR (AFR) irrep $Y_{1}^{-}$, as shown in Fig. 5. Using the INVARIANTS software package, we find that these four primary order parameters provide a quadrilinear invariant term in the free energy expansion, so that simultaneously invoking the framework $\left(Y_{3}^{+}\right)$and the cation $\left(\Gamma_{4}^{+}, Y_{1}^{-}\right)$rotational modes breaks the inversion symmetry of parent, thus allowing them to couple to a secondary (i.e., improper) ferroelectric moment $\left(\Gamma_{2}^{-}\right)$.

Because the $\mathrm{Pbl}_{4}$ framework is only slightly disturbed in switching between the $+P$ and $-P$ states, it is convenient to treat the $Y_{3}^{+}$framework rotation as a large preexisting structural feature in a slightly less-symmetric parent structure with space group Pnmm ( $\# 59, D_{2 h}^{13}$ ) and unit-cell basis and origin identical to those of the child. For this less-symmetric parent structure, the FR and AFR cation rotations form a simple trilinear invariant with the ferroelectric moment.

The $+P$ cation arrangement is obtained from the parent via the superposition of a $90^{\circ} \mathrm{FR}$ motion $\left(\Gamma_{4}^{+}\right)$and a $90^{\circ}$ AFR motion $\left(Y_{1}^{-}\right)$, so that two of the AMP cations are rotated by a full $180^{\circ}$ while the other two remain stationary (as shown in Fig. 1). The $-P$ cation arrangement is achieved instead by merely reversing the sense of the AFR contribution. Due to the unfavorable energy at the $90^{\circ}$ midpoint of rotation, it makes no sense to view the FR and AFR rotations as separate processes; they must occur simultaneously and cooperatively so that the $\Gamma_{4}^{+}$and $Y_{1}^{-}$order parameters are tightly coupled to have equal amplitudes. The product of two such large order parameters facilitates a strong trilinear coupling to the FE polarization. It remarkable that two centric modes, i.e., $\Gamma_{4}^{+}$and 
$Y_{1}^{-}$, combine together to give rise a hybrid mode, which in turn, breaks inversion symmetry and allows the polarization to arise in this system. Moreover, the coherent switching of both of them, i.e., the switching of the hybrid model, allows switching the polarization giving rise to a hidden pseudo-rotation in $k$-space, as highlighted in this work with the switchable Rashba anisotropy ellipse. It is intriguing that the switchable Rashba anisotropy occurs coherently at different relevant points in the $k$-space.

In summary, HOIPs are emerging as a class of photovoltaic materials. In this work, DFT methods have been used to study the ferroelectric properties and Rashba spin-orbit effect as well as the coupling between the FE polarization $\left(P_{s}\right)$ and the anisotropy of the Rashba parameter $\left(a_{R}\right)$ for a layered HOIP, (AMP)Pbl , where AMP is 4-(aminomethyl)piperidinium. We calculate $P_{s}$ and $a_{R}$ to be $10.72 \mu \mathrm{C} \mathrm{cm}^{-2}$ and $2.39 \mathrm{eV} \AA$, respectively, which are nicely consistent with the experimentally reported values of $9.8 \mu \mathrm{C} \mathrm{cm}^{-2}$ and $2.60 \mathrm{eV} \AA$. To gain insight into the origin of the polarization, the contributions of the organic $\mathrm{AMP}^{2+}$ cations $\left(10.34 \mu \mathrm{C} \mathrm{cm}^{-2}\right)$ and inorganic $\left[\mathrm{Pbl}_{4}\right]^{2-}$ framework $\left(0.59 \mu \mathrm{C} \mathrm{cm}^{-2}\right)$ have been disentangled, demonstrating that the AMP cations contribute almost all of the total polarization. This clearly suggests that a proper choice of organic cations could possibly enhance the ferroelectric polarization, by exploiting both the molecular dipole moment and their relative tilting, giving rise to an uncompensated electric polarization.

As previously reported for Rashba ferroelectrics, the sense of rotation of the spin-texture can be reversed by a switching of the FE polarization direction along the polar axis ${ }^{40}$. However, in (AMP)Pbl we calculate a large and robust anisotropy in the Rashba parameter. Remarkably, we find that the major and minor axes of the ellipse can be exchanged under reversal of the electric polarization, causing a $90^{\circ}$ pseudo-rotation of the Rashba anisotropy, which we have also been confirmed from a theoretical $k \cdot p$ model. The same effect is observed in both the VBs and CBs at multiple points in the BZ where the Rashba splitting is significant. A structural mechanism for this effect is presented and explained in terms of a quadrilinear coupling of order parameters involving two large-amplitude AMPcation rotation modes, a large octahedral framework rotation, and the ferroelectric polarization. To the best of our knowledge, this is the first report of a coupling of ferroelectric polarization and switchable highly anisotropic Rashba spin-split bands and it may be possible that the same property can be found in other layered hybrid perovskites. This deserves further investigation. Indeed, spinoptoelectronic devices based on hybrid organic-inorganic trihalide perovskites like spin-LEDs have been recently discussed for the parent compound $\mathrm{MAPbBr}_{3}{ }^{46}$, circularly polarized light detections have been considered for chiral hybrid perovskite ${ }^{47}$, spin-FETs. This strong coupling may provide a potential probe of the spin degrees of freedom in photovoltaic materials and a different avenue for developing spin-optoelectronic devices based on HOIPs materials.

\section{METHODS}

\section{Computational details}

The starting point for our calculations is the experimental crystallographic data reported in ref. ${ }^{33}$, where we relax all of the atoms of the ferroelectric structure until the Hellmann-Feynman forces are smaller than $0.001 \mathrm{eV}$ $\AA^{-1}$. The projector augmented wave (PAW) ${ }^{48}$ method is used to solve Kohn-Shan equations with the PBE exchange-correlation functional ${ }^{49}$, as implemented in the Vienna ab initio simulation package. The energy cutoff for the plane wave expansion is set to $550 \mathrm{eV}$, and a $2 \times 2 \times 2$ Monkhorst-Pack grid of $k$-point is used after systematic convergence tests. The van der Waals interactions are taken into account in calculations using the DFT-D3 method ${ }^{50}$. The Berry phase approach is used to evaluate the ferroelectric polarization by constructing a properly AFE reference state and a suitable path connecting FE with AFE states ${ }^{40,51,52}$. Details of this approach are discussed in the results section. Spin-orbit interaction is selfconsistently considered in all band structure calculations, but not in the calculations of polarization. Van der Waals interactions are included in all calculations $^{53}$

\section{DATA AVAILABILITY}

The data that support the findings of this study are available from the authors on reasonable request, see author contributions for specific data sets.

Received: 1 July 2020; Accepted: 27 October 2020;

Published online: 03 December 2020

\section{REFERENCES}

1. Cui, Y. et al. Over $16 \%$ efficiency organic photovoltaic cells enabled by a chlorinated acceptor with increased open-circuit voltages. Nat. Commun. 10, 2515 (2019).

2. Hong, L. et al. Eco-compatible solvent-processed organic photovoltaic pells with pver 16\% efficiency. Adv. Mater. 31, e1903441 (2019).

3. Aguiar, A. et al. Simple BODIPY dyes as suitable electron-donors for organic bulk heterojunction photovoltaic cells. Dyes Pigments 172, 107842 (2020).

4. Tsai, $H$. et al. High-efficiency two-dimensional Ruddlesden-Popper perovskite solar cells. Nature 536, 312-316 (2016).

5. Wu, T. \& Wang, J. Global discovery of stable and non-toxic hybrid organicinorganic perovskites for photovoltaic systems by combining machine learning method with first principle calculations. Nano Energy 66, 104070 (2019).

6. Ghosh, D., Aziz, A., Dawson, J. A., Walker, A. B. \& Islam, M. S. Putting the squeeze on lead iodide perovskites: pressure-induced effects to tune their structural and optoelectronic behavior. Chem. Mater. 31, 4063-4071 (2019).

7. Ferdani, D. W. et al. Partial cation substitution reduces iodide ion transport in lead iodide perovskite solar cells. Energy Environ. Sci. 12, 2264-2272 (2019).

8. Brenes, R., Eames, C., Bulovic, V., Islam, M. S. \& Stranks, S. D. The impact of atmosphere on the local luminescence properties of metal halide perovskite grains. Adv. Mater. 30, e1706208 (2018)

9. Kahmann, S. \& Loi, M. A. Hot carrier solar cells and the potential of perovskites for breaking the Shockley-Queisser limit. J. Mater. Chem. C 7, 2471-2486 (2019)

10. Wang, Z., Song, Z., Yan, Y., Liu, S. \& Yang, D. Perovskite-a perfect top cell for tandem devices to break the S-Q limit. Adv. Sci. 6, 1801704 (2019).

11. Fang, H.-H., Adjokatse, S., Shao, S., Even, J. \& Loi, M. A. Long-lived hot-carrier light emission and large blue shift in formamidinium tin triiodide perovskites. Nat. Commun. 9, 243 (2018).

12. Polman, A., Knight, M., Garnett, E. C., Ehrler, B. \& Sinke, W. C. Photovoltaic materials: present efficiencies and future challenges. Science 352, aad4424 (2016)

13. Tayebjee, M. J., McCamey, D. R. \& Schmidt, T. W. Beyond Shockley-Queisser: molecular approaches to high-efficiency photovoltaics. J. Phys. Chem. Lett. 6 2367-2378 (2015).

14. Shi, C. et al. Two-dimensional organic-inorganic hybrid rare-earth double perovskite ferroelectrics. J. Am. Chem. Soc. 142, 545-551 (2020).

15. Pandey, R. et al. Mutual insight on ferroelectrics and hybrid halide perovskites: a platform for future multifunctional energy conversion. Adv. Mater. 31, e1807376 (2019).

16. Balachandran, P. V., Kowalski, B., Sehirlioglu, A. \& Lookman, T. Experimental search for high-temperature ferroelectric perovskites guided by two-step machine learning. Nat. Commun. 9, 1668 (2018).

17. Hua, X.-N. et al. A room-temperature hybrid lead iodide perovskite ferroelectric. J. Am. Chem. Soc. 140, 12296-12302 (2018).

18. Pan, Q. et al. A three-dimensional molecular perovskite ferroelectric: (3-ammoniopyrrolidinium)RbBr3. J. Am. Chem. Soc. 139, 3954-3957 (2017).

19. Xu, W.-J. et al. A molecular perovskite with switchable coordination bonds for hightemperature multiaxial ferroelectrics. J. Am. Chem. Soc. 139, 6369-6375 (2017).

20. Lee, H., Im, J. \& Jin, H. Emergence of the giant out-of-plane Rashba effect and tunable nanoscale persistent spin helix in ferroelectric SnTe thin films. Appl. Phys. Lett. 116, 022411 (2020).

21. Lin, Z., Si, C., Duan, S., Wang, C. \& Duan, W. Rashba splitting in bilayer transition metal dichalcogenides controlled by electronic ferroelectricity. Phys. Rev. B 100, 155408 (2019).

22. He, J. et al. Tunable metal-insulator transition, Rashba effect and Weyl Fermions in a relativistic charge-ordered ferroelectric oxide. Nat. Commun. 9, 492 (2018).

23. Djani, $\mathrm{H}$. et al. Rationalizing and engineering Rashba spin-splitting in ferroelectric oxides. npj Quantum Mater. 4, 51 (2019).

24. Kepenekian, M. et al. Rashba and Dresselhaus effects in hybrid organic-inorganic perovskites: from basics to devices. ACS Nano 9, 11557-11567 (2015).

25. Zhai, Y. et al. Giant Rashba splitting in 2D organic-inorganic halide perovskites measured by transient spectroscopies. Sci. Adv. 3, e1700704 (2017).

26. Picozzi, S. Ferroelectric Rashba semiconductors as a novel class of multifunctional materials. Front. Phys. 2, 10 (2014).

27. Vajna, S. et al. Higher-order contributions to the Rashba-Bychkov effect with application to the Bi/Ag(111) surface alloy. Phys. Rev. B 85, 075404 (2012). 
28. Maaß, H. et al. Spin-texture inversion in the giant Rashba semiconductor BiTel. Nat. Commun. 7, 11621 (2016).

29. Kim, M., Im, J., Freeman, A. J., Ihm, J. \& Jin, H. Switchable $S=1 / 2$ and J $=1 / 2$ Rashba bands in ferroelectric halide perovskites. Proc. Natl. Acad. Sci. USA 111, 6900-6904 (2014).

30. Rinaldi, C. et al. Ferroelectric control of the spin texture in GeTe. Nano Lett. 18, 2751-2758 (2018).

31. Krempaský, J. et al. Entanglement and manipulation of the magnetic and spinorbit order in multiferroic Rashba semiconductors. Nat. Commun. 7, 13071 (2016).

32. Park, I. H. et al. Ferroelectricity and Rashba effect in a two-dimensional DionJacobson hybrid organic-inorganic perovskite. J. Am. Chem. Soc. 141, 15972-15976 (2019).

33. Zheng, F., Takenaka, H., Wang, F., Koocher, N. Z. \& Rappe, A. M. First-principles calculation of the bulk photovoltaic effect in $\mathrm{CH}_{3} \mathrm{NH}_{3} \mathrm{Pbl}_{3}$ and $\mathrm{CH}_{3} \mathrm{NH}_{3} \mathrm{Pbl}_{3-\mathrm{x}} \mathrm{Cl}_{x}$.J. Phys. Chem. Lett. 6, 31-37 (2015).

34. Li, L. et al. Two-dimensional hybrid perovskite-type ferroelectric for highly polarization-sensitive shortwave photodetection. J. Am. Chem. Soc. 141, 2623-2629 (2019).

35. von Hippel, A. Ferroelectricity, domain structure, and phase transitions of Barium Titanate. Rev. Mod. Phys. 22, 221-237 (1950).

36. Shieh, J., Yeh, J. H., Shu, Y. C. \& Yen, J. H. Hysteresis behaviors of barium titanate single crystals based on the operation of multiple $90^{\circ}$ switching systems. Mater. Sci. Eng. B 161, 50-54 (2009).

37. Fonseca Guerra, C., Handgraaf, J.-W., Baerends, E. J. \& Bickelhaupt, F. M. Voronoi deformation density (VDD) charges: assessment of the Mulliken, Bader, Hirshfeld, Weinhold, and VDD methods for charge analysis. J. Comput. Chem. 25, 189-210 (2004).

38. Reed, A. E., Weinstock, R. B. \& Weinhold, F. Natural population analysis. J. Chem. Phys. 83, 735-746 (1985).

39. Frisch, M. J. et al. Gaussian 16, Revision A.03 (Wallingford, CT, 2016).

40. Spaldin, N. A. A beginner's guide to the modern theory of polarization. J. Solid State Chem. 195, 2-10 (2012).

41. Rabe, K. M., Ahn, C., \& Triscone, J.-M. Physics of Ferroelectrics: A Modern Perspective (Springer, Berlin, Heidelberg, 2007)

42. Di Sante, D., Barone, P., Bertacco, R. \& Picozzi, S. Electric control of the giant Rashba effect in bulk GeTe. Adv. Mater. 25, 3625-3626 (2013).

43. Stroppa, A. et al. Tunable ferroelectric polarization and its interplay with spinorbit coupling in tin iodide perovskites. Nat. Commun. 5, 5900 (2014).

44. Tao, L. L., Paudel, T. R., Kovalev, A. A. \& Tsymbal, E. Y. Reversible spin texture in ferroelectric HfO2. Phys. Rev. B 95, 245141 (2017).

45. Tao, L. L. \& Tsymbal, E. Y. Persistent spin texture enforced by symmetry. Nat. Commun. 9, 2763 (2018).

46. Wang, J. et al. Spin-optoelectronic devices based on hybrid organic-inorganic trihalide perovskites. Nat. Commun. 10, 129 (2019).

47. Chen, $C$. et al. Circularly polarized light detection using chiral hybrid perovskite. Nat. Commun. 10, 1927 (2019).

48. Blöchl, P. E. Projector Augmented-wave method. Phys. Rev. B 50, 17953-17979 (1994).

49. Perdew, J. P. et al. Restoring the density-gradient expansion for exchange in solids and surfaces. Phys. Rev. Lett. 100, 136406 (2008).

50. Grimme, S., Antony, J., Ehrlich, S. \& Krieg, H. A consistent and accurate ab initio parametrization of density functional dispersion correction (DFT-D) for the 94 elements H-Pu. J. Chem. Phys. 132, 154104 (2010).

51. Resta, R. Macroscopic polarization in crystalline dielectrics: the geometric phase approach. Rev. Mod. Phys. 66, 899-915 (1994).

52. King-Smith, R. D. \& Vanderbilt, D. Theory of polarization of crystalline solids. Phys. Rev. B 47, 4 (1993).

53. Grimme, S. Accurate description of van der Waals complexes by density functional theory including empirical corrections. J. Comput. Chem. 25, 1463-1473 (2004).

\section{ACKNOWLEDGEMENTS}

This project has received funding from the European Union's Horizon 2020 research and innovation program under the Marie Skłodowska-Curie grant agreement No. 713679 and from the Universitat Rovira i Virgili (URV). J.M.P. and C.d.G thank the Spanish Ministry of Science (grants CTQ2017-87269-P and CTQ2017-83566-P) and the Generalitat de Catalunya (grant 2017SGR629) for support. J.M.P. also thanks ICREA foundation for an ICREA ACADEMIA award. H.G. acknowledges support from the National Postdoctoral Program for Innovative Talents (No. BX20190361) and Guangdong Basic and Applied Basic Research Foundation (No. 2019A1515110965). B.J.C. acknowledges helpful conversations with Harold T. Stokes and John Colton. B.J. C. and A.S. thank Z. V. Vardeny for useful discussions. A.S. acknowledges A. Cassinese, F. Chiarella, and M. Barra for useful discussions. F.W. acknowledges the kind hospitality by CNR-SPIN c/o Department of Chemical and Physical Science of University of L'Aquila (Italy) where this project was discussed during the vising period from November 2, 2019 to December 14, 2019.

\section{AUTHOR CONTRIBUTIONS}

A.S. conceived and designed the study; A.S., C.G., and J.M.P. supervised the project; F.W. performed the DFT calculations and drafted the paper; H.G. constructed the $k \cdot p$ model and assisted the analysis of Rashba anisotropy. B.J.C. conducted the symmetry-mode analysis. All authors reviewed and approved the paper.

\section{COMPETING INTERESTS}

The authors declare no competing interests.

\section{ADDITIONAL INFORMATION}

Supplementary information is available for this paper at https://doi.org/10.1038/ s41524-020-00450-z.

Correspondence and requests for materials should be addressed to C.d.G. or A.S.

Reprints and permission information is available at http://www.nature.com/ reprints

Publisher's note Springer Nature remains neutral with regard to jurisdictional claims in published maps and institutional affiliations.

\begin{abstract}
Open Access This article is licensed under a Creative Commons Attribution 4.0 International License, which permits use, sharing, adaptation, distribution and reproduction in any medium or format, as long as you give appropriate credit to the original author(s) and the source, provide a link to the Creative Commons license, and indicate if changes were made. The images or other third party material in this article are included in the article's Creative Commons license, unless indicated otherwise in a credit line to the material. If material is not included in the article's Creative Commons license and your intended use is not permitted by statutory regulation or exceeds the permitted use, you will need to obtain permission directly from the copyright holder. To view a copy of this license, visit http://creativecommons. org/licenses/by/4.0/.
\end{abstract}

(c) The Author(s) 2020 